\title{
Spectroscopic analysis for polarization sensitive optical coherent tomography
}

\author{
Marcin R. Strąkowski ${ }^{*}$ Jerzy Pluciński, and Bogdan B. Kosmowski \\ Department of Optoelectronics and Electronic Systems, Faculty of Electronics, Telecommunications and \\ Informatics, Gdańsk University of Technology, Narutowicza 11/12, 80-233 Gdańsk, Poland
}

Received May 17, 2011; accepted September 27, 2011; published September 30, 2011

\begin{abstract}
Polarization Sensitive Optical Coherent Tomography (PSOCT) is a novel optical method to examine a broad range of scattering materials. PS-OCT is an extension of OCT systems, which enables cross-sectional visualization of the device inner structure, as well as analysis of the polarization state of light backscattered from particular points inside the tested device. Polarization sensitive analysis is a very useful tool in OCT measurements. However, it brings a number of problems referring to measurement signal processing, birefringence changes of the investigated sample over the spectral width of a broadband light source. In this paper these problems have been discussed and possible solutions have been given.
\end{abstract}

Optical coherence tomography (OCT) is an interferometric method for two and three-dimensional imaging of surface and subsurface structure of scattering materials with micrometer resolution. The OCT measurements are performed in a non-contact and nondestructive way, therefore, this method is totally safe for the tested object [1-2]. Presently, the main applications of OCT cover medical diagnosis in the field of ophthalmology, dermatology and also dentistry [3-4]. However, the usefulness of OCT in beyond medical applications has been noticed. Nowadays, OCT is used in industry and science for material characterization, surface and subsurface defect detection, strain fields mapping inside polymer materials, ceramic materials examination, as well as art conservation [5-9].

OCT is based on the interference of light beams which have low temporal coherence (WLI - White Light Interferometry or LCI - Low-Coherence Interferometry). Thereby, light backscattered from particular scattering points inside the investigated object can be selectively detected, which is needed for tomography imaging $[1,2$, 10]. There are several types of OCT systems. Our research has been concentrated on Polarization Sensitive Optical Coherent Tomography (PS-OCT). Polarization sensitive analysis provides unique benefits to OCT measurements, which allows studying phenomena occurring in a tested sample that cannot be investigated by conventional OCT methods. This measurement method gives structural information about an investigated sample

${ }^{*}$ E-mail: marcin.strakowski@eti.pg.gda.pl and also enables selective visualization of an optical anisotropic structure [1, 2, 7]. Hence, we apply this method to investigate birefringent complex structures like liquid crystals cells, polymer retarders, as well as polymer and ceramics composites. The PS-OCT system, which has been designed in the Gdańsk University of Technology (Department of Optoelectronics and Electronic Systems), has been shown in Fig. 1 and Fig. 2.

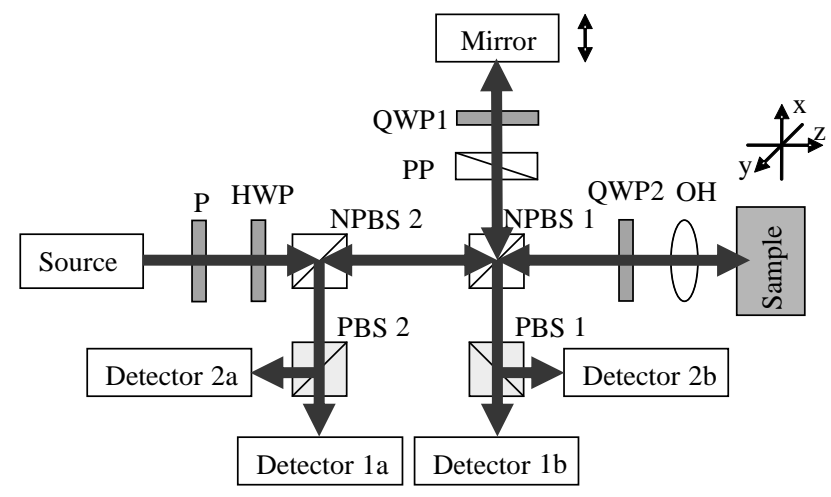

Fig. 1. The PS-OCT system setup; NPBS $1 \div 2$ - non-polarizing beamsplitter, PBS $1 \div 2$ - polarizing beamsplitter $\mathrm{P}$ - polarization plate, QWP $1 \div 2$ - quarter-wave plate, HWP - half-wave plate.

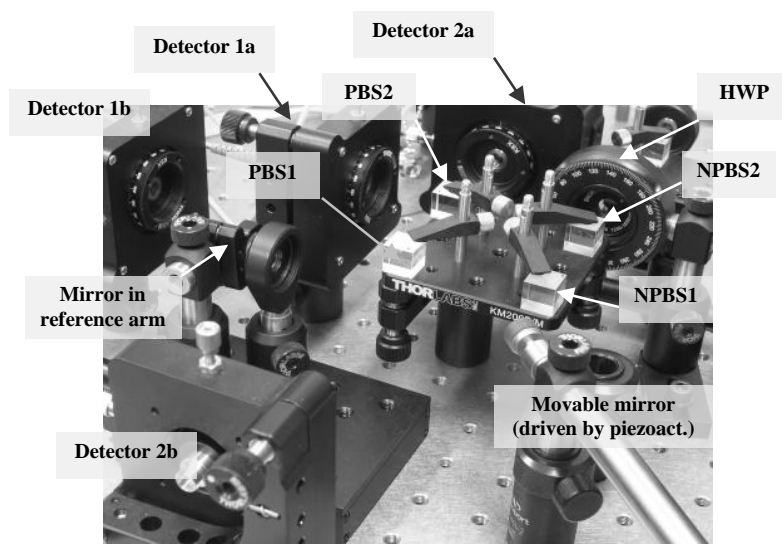

Fig. 2. The optical part of the PS-OCT system. 
The PS-OCT system (Figs. 1-2) is based on the Michelson interferometer where the movable mirror driven by a piezo actuator is placed in the reference arm. The interferometer is illuminated by a broadband supercontinuum light source. The backscattered light beams from the sample and light reflected from the mirror are recombined and subsequently separated by a polarization beam splitter into orthogonal components which are recorded by two pairs of photodetectors (in Fig.1: Detector 1a and 1b, Detector $2 \mathrm{a}$ and $2 \mathrm{~b}$ ). These detectors provide polarization diversity detection with excess noise elimination. The details of the developed system have been described in [2, 10, 11]. In this system, as well as others such as PS-OCT systems, the polarization state analysis is based on Jones formalism. The birefringent parameters of the investigated sample are calculated from the magnitude and phase of a received interference signal. These calculations are based on Eqs. (1)-(2):

$$
\begin{array}{r}
\Gamma(z)=a \tan \left(\frac{\left|I_{H}(z)\right|}{\left|I_{V}(z)\right|}\right), \\
\Theta(z)=\frac{\pi-\Delta \varphi(z)}{2},
\end{array}
$$

where: $\mathrm{I}_{\mathrm{H}}(z)$ and $\mathrm{I}_{\mathrm{V}}(z)$ - the recorded interference signals at detector 1 and detector 2 , respectively, $\Delta \varphi(z)-$ the phase difference between recorded interference signals, $\Gamma(z)$ - the depth $(z)$ profile of retardation angle changes inside the tested device, $\Theta(z)$ - the depth profile of an optical axis orientation of the device.

This method of polarization sensitive analysis has been developed for the PS-OCT systems where the light source fulfills the condition given by Eq. (3):

$$
\lambda_{0}>\Delta \lambda
$$

where: $\lambda_{0}-$ central wavelength and $\Delta \lambda$ - bandwidth of the light source.

In this particular case, both $\Gamma(z)$ and $\Theta(z)$ characteristics are calculated for the central wavelength with sufficient accuracy. However, the range of measured values of the retardation angle and fast axis orientation is limited to $90^{\circ}$ for $\Gamma(z)$ and $180^{\circ}$ for $\Theta(z)$. For high birefringent materials like liquid crystals cells or grass-fiber reinforced polymers the absolute values of $\Gamma(z)$ can be difficult to calculate [6] Great progress in ultra-broadband light sources results in obtaining not only a better resolution of OCT measurements but also opens up new possibilities for successful application of spectroscopic analysis. Based on those well known methods, a new method for ultra-high resolution PS-OCT systems can be developed [11]. This is a new approach in PS-OCT, which enables polarization sensitive analysis to be performed over the spectral range of a broadband light source. The method is based on spectroscopic analysis of the spectral characteristic of the light which is backscattered from particular points inside the investigated device. One of the most important problems which must be solved is the selective reconstruction of the spectral characteristics from recorded interference signals. Consider the equation:

$$
\begin{aligned}
& S_{D}(k)=(D C)+ \\
& +2 \cdot \sum_{n=1}^{N}\left|S_{R n}(k)\right| \cdot \cos \left(2 k \cdot\left(z_{R}-z_{n}\right)+\arg \left\{S_{R n}(k)\right\}\right)+ \\
& +(\text { auto }- \text { correlation terms })
\end{aligned}
$$

which describes an OCT measurement signal in the spectral domain. The $S_{R n}(k)$ is the cross-spectral density of the interfering beams. It depends on the spectral characteristics of the light from the measurement and reference arm of a low-coherent interferometer. By the use of the Fourier transform (FT) it is possible to transfer the $S_{D}(k)$ function to the time domain, which has been shown as relation (5).

$$
\begin{aligned}
& F T\left\{S_{D}(k)\right\}=I_{D}(z)=(\text { DC terms })+ \\
& +\sum_{n=1}^{N} G_{R n}(z) \otimes\left(\delta\left(z \pm 2\left(z_{R}-z_{n}\right)\right)\right)+ \\
& +(\text { auto }- \text { correlation terms })
\end{aligned}
$$

where: $G_{R n}(z)$ - the cross-correlation function of the light from the reference arm and backscattered from a particular $\mathrm{n}$ point inside the investigated sample, $\left(z_{R}-z_{n}\right)$ determines the optical delay between interfering beams. The cross-correlation function $G_{R n}(z)$ and cross-spectral density $S_{R n}(k)$ are related to each other by the pair of Fourier transforms. Therefore, if the $G_{R n}(z)$ function can be obtained, the $S_{R n}(k)$ will be determined. According to Eq. (5), the $G_{R n}(\mathrm{z})$ function is separated in the time domain, which facilitates its extraction from $I_{D}(z)$ characteristic. Based on the described relations the measurement signal procedure can be developed in order to obtain the complex spectral characteristic of the backscattered light [12-14]. An example of the signal processing which has been described in details in [12-13], has been shown in Fig. 3 .

The presented algorithm can be easily adopted to our time domain PS-OCT system. The real part of $I_{D}(z)$ is proportional to the time domain OCT measurement signal. Therefore using a Hilbert transform and continuing the procedure for $I_{D}(z)$ it is possible to obtain the spectral information. This algorithm was used for $I_{H}(z)$ and $I_{V}(z)$ signals processing. The spectral characteristics of retardation angle changes were calculated based on the Eqs. (1) and (2) performed for each wavelength value. An 
example of measurement results obtained for an optical retarder has been presented in Fig. 4.

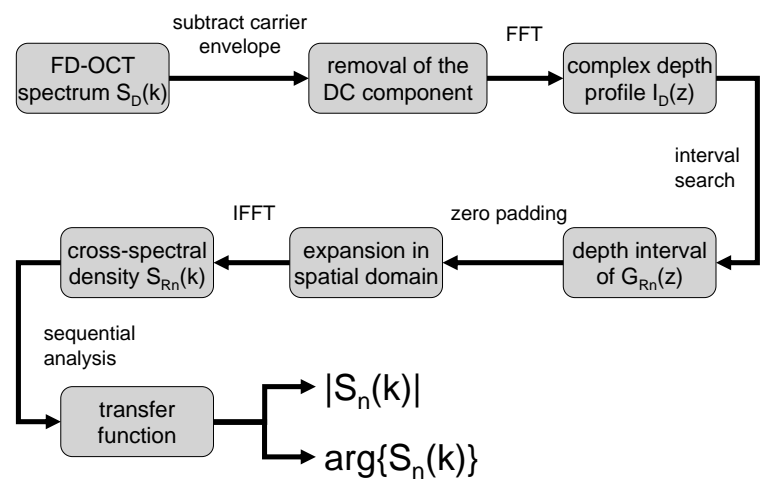

Fig. 3. The diagram of measurement signal processing in optical coherence tomography with spectroscopic analysis; $S_{n}(k)$ - complex spectral characteristic of backscattered light from $n$ point inside the device under tests.

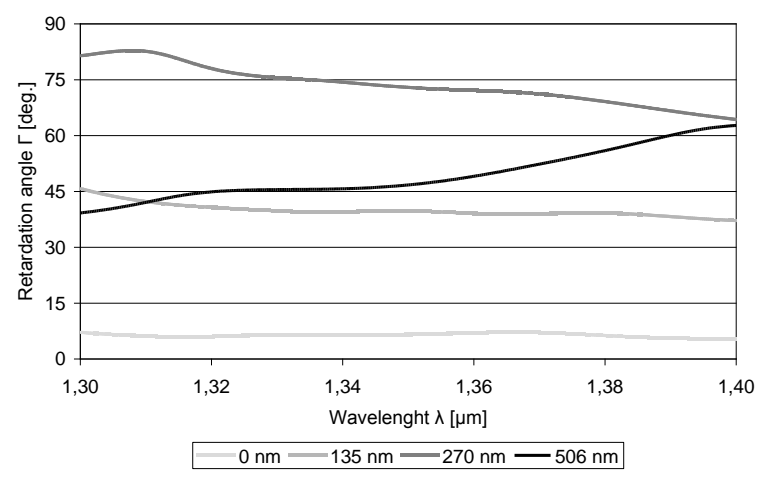

Fig. 4. Measured spectral characteristic of the retardation angle of the optical retarder.

As the device under test the Soleil-Babinet compensator was used. The retardation of the device was set to 0 , $13 \mathrm{~nm}, 270 \mathrm{~nm}$ and $506 \mathrm{~nm}$. These values correspond to $0^{\circ}$, $36^{\circ}, 72^{\circ}$ and $135^{\circ}$ of the retardation angle for a wavelength equal of $1.35 \mu \mathrm{m}$. The measurements were carried out using the PS-OCT system presented in Figs. 12 , and described in $[2,10,11]$. As expected, the growth of the retardation value caused an increase in the mean value and slope of the measured retardation characteristic. If the retardation angle exceeds the $90^{\circ}$, the direction of the slope of the measured characteristics is changing. Moreover, with continuous growth of the retardation value the measured mean value of the retardation angle decreases. Therefore, by analyzing the slope and mean value of the retardation angle spectral characteristic it is possible to determine the absolute values of the investigated sample retardation.
In conclusion, the spectroscopic analysis can be a useful method in polarization sensitive optical coherence tomography. It brings unique benefits to PS-OCT measurement, delivering full spectral information about backscattered light from particular points inside the tested device. It enables investigation of optical anisotropy changes occurring inside the device in the range of the broadband light source spectrum. Moreover, the combination of PS-OCT and spectroscopic analysis enables to conduct optical anisotropy and spectroscopic measurements simultaneously. Therefore, polarization sensitive optical coherence tomography combined with spectroscopic analysis can be an interesting tool for the investigation and quality assessment of complex objects like printed electronic parts or polymer composite materials.

This study was partially supported by the Polish Ministry of Science and Higher Education under the grant No. N N515 335636, and DS programs of the Faculty of Electronics, Telecommunications and Informatics, Gdańsk University of Technology.

\section{References}

[1] A.F. Fercher, W. Drexler, C.K. Hitzenberg, Rep. Prog. Phys. 66, 239 (2003).

[2] M.R. Strąkowski, J. Pluciński, M. Jędrzejewska-Szczerska, R. Hypszer, M. Maciejewski, B.B. Kosmowski, Sens. and Actuat. A 142, 104 (2008).

[3] A. Szkulmowska, M. Szkulmowski, A. Kowalczyk, M. Wojtkowski, Opt. Lett 33, 1425 (2008).

[4] M. Wojtkowski, T. Bajraszewski, P. Targowski, A. Kowalczyk, Opt. Lett. 28, 1745 (2003).

[5] D. Stifter, P. Burgholzer, O. Höglinger, E. Götzinger, C.K. Hitzenberger, Appl. Phys. 76, 947 (2003).

[6] D. Stifter, Appl. Phys. B 88, 337 (2007).

[7] D. Stifter, K. Wiesauer, M. Wurm, E. Schlotthauer, J. Kastner, M. Pircher, E. Götzinger, C.K. Hitzenberger, Meas. Sci. Technol. 19, 074011 (2008).

[8] K. Wiesauer, M. Pirchen, E. Gotzinger, C.K. Hitzenberger, Comp. Science Techn. 67, 3051 (2007).

[9] P. Targowski, B. Rouba, M. Góra, L. Tymańska-Widmer, J. Marczak, A. Kowalczyk, Appl. Phys. A 92, 1 (2008).

[10] J. Pluciński, R. Hypszer, P. Wierzba, M. Strąkowski, M. JędrzejewskaSzczerska, M. Maciejewski, B.B. Kosmowski, Bulletin of the Polish Academy of Sciences - Technical Sciences 56, 155 (2008).

[11] M. Strąkowski, Analiza stanu polaryzacji światta w uktadach optycznej tomografii koherentnej dla badań struktury materiałów optoelektronicznych i mikroelektronicznych ( $\mathrm{PhD}$ Dissertation, WETI PG 2010).

[12] Ch. Kasseck, V. Jaedicke, N.C. Gerhardt, H. Welp, M.R. Hofmann, Opt. Com. 283, 4816 (2010).

[13] Ch. Kasseck, V. Jaedicke, N.C. Gerhardt, H. Welp, M.R. Hofmann, Proc. of SPIE 7554, 75542T (2010).

[14] G. Latour, J. Moreau, M. Elias, J-M. Frigerio, Opt. Com. 283, 4810 (2010). 